\title{
50 Jahre Deutsche Gesellschaft für Transfusionsmedizin und Immunhämatologie (DGTI)
}

Im Herbst 2004 kann die «Deutsche Gesellschaft für Transfusionsmedizin und Immunhämatologie» (DGTI) auf ihr 50jähriges Bestehen zurück blicken. Am 30. Oktober 1954 war bei einem Treffen von Repräsentanten der am Thema Bluttransfusion interessierten medizinischen Disziplinen in Bad Homburg v.d.H. die «Deutsche Gesellschaft für Bluttransfusion» gegründet worden, die 1973 in «Deutsche Gesellschaft für Bluttransfusion und Immunhämatologie» umbenannt wurde. Seit 1986 führt sie den Namen «Deutsche Gesellschaft für Transfusionsmedizin und Immunhämatologie» (DGTI).

Dieses Jubiläum bietet Anlass, in einem Sonderheft unserer Zeitschrift TRANSFusion MEdicine AND HeMOTHERAPY unser Fachgebiet einmal selbst zum Gegenstand wissenschaftlicher Reflexion zu machen. Anders als in der klinischen Forschung verwendet die hier eingesetzte Methode aber kein prospektives, randomisiertes und doppelt verblindetes Studiendesign, da dieses Mal nicht naturgesetzliche, biologische Prozesse zu untersuchen sind. Stattdessen haben die Autoren der folgenden Beiträge ihr jeweiliges Thema mit den Mitteln der Medizingeschichte in Form einer retrospektiven historischen Darstellung und Analyse erarbeitet.

Nicht nur Menschen haben ihre individuelle, unwiederholbare Geschichte, die in der historischen Gattung der Biografie ihre literarische und wissenschaftliche Gestaltung findet. Auch Institutionen - wie etwa eine medizinische Fachgesellschaft weisen eine unverwechselbare geschichtliche Entwicklung auf. Darin spiegeln sich nicht nur die Lebensläufe der beteiligten Akteure mit ihren oft divergierenden Zielen, ihren Erfolgen und ihrem Scheitern wider, sondern auch die politischen, gesellschaftlichen und wissenschaftlichen Zeitumstände, in denen die jeweils handelnden Personen standen. Es ist eine wesentliche Aufgabe der Medizingeschichte, nicht nur die Zielgerichtetheit, sondern ebenso auch die Kontingenz historischer Ereignisse, Prozesse und Strukturen darzustellen, die in ihrem konkreten Auftreten zwar möglich, aber weder notwendig noch beliebig sind.
Da eine umfassende medizinhistorische Darstellung der jüngeren Geschichte von Transfusionsmedizin und Immunhämatologie in Form einer Monografie bislang noch aussteht, haben sich die Herausgeber dazu entschlossen, im Rahmen dieses Sonderheftes zunächst einmal eine Dokumentation von Ereignissen, Prozessen und Institutionen zu erarbeiten, die als eine erste Bestandsaufnahme der Fachgeschichte dienen soll. Die Autoren, die wir für diese aufwändige und anspruchsvolle Aufgabe gewinnen konnten, sind keine professionellen Medizinhistoriker sondern profilierte Repräsentanten der Transfusionsmedizin und Immunhämatologie. Dass die von Beginn an als deutschsprachig verstandene Gesellschaft in ihrer Entwicklung durch die gesundheitspolitischen Rahmenbedingungen in mehreren Staaten mitgeprägt wurde, macht die Darstellung umso facettenreicher.

Einige dieser Kolleginnen und Kollegen, die auf eine lange Berufstätigkeit zurück blicken können, beschreiben die Entwicklung des Faches bzw. seiner Teilgebiete zugleich aus der Perspektive aktiv an den geschilderten Vorgängen beteiligter Zeitzeugen. Dabei ergibt sich - bei allem Bemühen um Unparteilichkeit - notwendiger Weise auch ein subjektiver Blickwinkel, aus dem heraus die Darstellung ihre Lebendigkeit und ihren Spannungsbogen erhält. Andere Autoren legen dem gegenüber den Schwerpunkt ihrer Beiträge mehr auf die Darlegung jener institutionellen Strukturen, in und zu denen sich das Fach im Verlauf des 20. Jahrhunderts, insbesondere während dessen zweiter Hälfte, entwickelt hat. Die auf diese Weise zusammen getragene breite Palette historischer Aspekte soll unseren Leserinnen und Lesern nicht nur einen geschichtlichen Leitfaden zur Entwicklung des Faches Transfusionsmedizin und Immunhämatologie liefern, sondern ihnen zugleich ein Medium der kritischen Reflexion über den aktuellen Standort der Disziplin bieten. Dafür enthalten die Arbeiten unserer Autoren zahlreiche Ansatzpunkte.

\section{KARGER}

(c) 2004 S. Karger GmbH, Freiburg

Fax +497614520714 
Einleitend wird die wechselvolle Geschichte der heutigen «Deutschen Gesellschaft für Transfusionsmedizin und Immunhämatologie» (DGTI) seit 1954 dargestellt, die den chronologischen Anlass für unser diesjähriges Jubiläum liefert. Der Beitrag über die Entstehung der Transfusionsmedizin seit dem Beginn des 20. Jahrhunderts belegt, dass die Gründung einer Fachgesellschaft für Bluttransfusion im Jahre 1954 kein spontaner Akt, sondern letztlich das Resultat einer langen historischen Entwicklung war, zu der Fachleute aus zahlreichen medizinischen Einzeldisziplinen ihren Beitrag geleistet haben. Mehrere Autoren beschäftigen sich mit den verschiedenen organisatorischen Strukturen, die sich in der Bundesrepublik Deutschland nach dem Ende des Zweiten Weltkrieges im Bereich der Transfusionsmedizin herausgebildet haben. Die Blutspendedienste des Deutschen Roten Kreuzes (DRK), die Staatlichen und Kommunalen Blutspendedienste (StKB) und die transfusionsmedizinischen Einrichtungen der Bundeswehr haben ihre jeweils eigene Geschichte wie auch ihre eigenständigen Aufgabenfelder, die sich jedoch der Natur der Sache gemäß in komplexer Weise berühren und zum Teil sogar überschneiden.

Der Beitrag über die Entwicklung der Transfusionsmedizin in der DDR legt dar, dass in dem von 1949 bis 1990 eigenstaatlichen östlichen Teil der heutigen Bundesrepublik Deutschland - trotz dessen für zahllose Menschen leidvoller systembedingter Trennung vom westlichen Deutschland und ungeachtet der politisch bedingten Abschottung vom als «kapitalistisch» gebrandmarkten westlichen Ausland - ein auf vergleichsweise hohem Niveau funktionsfähiges transfusionsmedizinisches Netzwerk entstand.

Die jeweils eigenständige Entwicklung der Transfusionsmedizin in Österreich und in der Schweiz nach 1945 wird in zwei Beiträgen detailliert dargestellt. Gerade der Vergleich der Entwicklung in den beiden Alpenländern mit jener in den zwei deutschen Staaten belegt jenes nicht unerhebliche Aus$\mathrm{maß}$ an Kontingenz, das bei prinzipiell ähnlichen Aufgaben in der Krankenversorgung $\mathrm{zu}$ doch recht unterschiedlichen Wegen in Aufbau und Organisation der transfusionsmedizinischen Strukturen geführt hat. Die Erfahrungen mit den divergenten Institutionen in vier staatlichen Ordnungen können durchaus dazu anregen, das jeweils eigene System immer wie- der kritisch auf seine Funktionsfähigkeit und deren Optimierung hin zu überprüfen.

Mit dem langen und mühsamen, aber letztendlich erfolgreichen Weg zum Facharzt für Transfusionsmedizin in Deutschland, wozu die bereits etablierte Gebietsbezeichnung in der DDR hilfreich beigetragen hat, befasst sich ein weiterer Beitrag.

In dem Artikel über Forschung und Lehre in der Transfusionsmedizin wird die akademische Etablierung des jungen Faches an den Medizinischen Fakultäten der Universitäten und Medizinischen Hochschulen beschrieben und auf die ökonomischen Zwänge der Fakultäten eingegangen, die gegenwärtig eine besondere Anstrengung zum Erhalt der Lehrstühle an den Universitäten erfordern. Zur Orientierung und Profilierung möge auch dieses Kompendium beitragen.

Mit dem unverzichtbaren normativen rechtlichen Rahmen, in den ein biomedizinisch wie medizinethisch höchst sensibles Gebiet wie die Transfusionsmedizin innerhalb einer rechtsstaatlichen Ordnung eingebettet werden muss, befassen sich drei Beiträge sachkompetenter Autoren.

Zum Schluss wird das hoch komplexe Arbeitsfeld des modernen Transfusionsmediziners zwischen traditioneller Blutspende und innovativer Biotechnologie beschrieben. Die Darstellung zeigt auf, wie sich die Transfusionsmedizin in den vergangenen Jahrzehnten nicht nur als eine eigenständige Disziplin etabliert, sondern auch in fast allen Teilaspekten gewandelt hat.

Die Herausgeber würden es begrüßen, wenn dieses Sonderheft zu einer fruchtbaren und dialogischen Auseinandersetzung möglichst vieler Kolleginnen und Kollegen mit Geschichte, Gegenwart und Zukunft des Fachgebietes beitragen könnte. Das 50-jährige Jubiläum der DGTI soll nicht nur zum historischen Rückblick anregen, es kann ebenso zum Ausblick in die kommenden Jahre ermutigen. Angesichts der äußerst kritischen ökonomischen Perspektive unseres bislang überwiegend solidarisch finanzierten Gesundheitswesens liegen hier auch fachpolitisch schwierige Aufgaben vor allen Beteiligten, die mit Kreativität und Augenmaß gemeistert werden müssen.

Norbert Müller, Essen Harald Klüter, Mannheim Axel W. Bauer, Heidelberg 